\title{
Treatment patterns and patient characteristics among early initiators of PCSK9 inhibitors
}

This article was published in the following Dove Press journal: Vascular Health and Risk Management

\section{Dionne M Hines' \\ Pallavi Rane ${ }^{2}$ \\ Jeetvan Patel ${ }^{2}$ \\ David J Harrison ${ }^{2}$ \\ Rolin L Wade'}

'Health Economics and Outcomes Research, IQVIA, Plymouth Meeting, PA, USA; ${ }^{2}$ Global Health Economics, Amgen Inc., Thousand Oaks, CA, USA
Correspondence: Pallavi Rane Global Health Economics, Amgen Inc., I Amgen Center Drive, Thousand Oaks, CA 91320-1799, USA

$\mathrm{Tel}+\mathrm{I} 8054909430$

Email prane@amgen.com
Purpose: To describe patient characteristics and treatment patterns among early initiators of proprotein convertase subtilisin/kexin type nine inhibitors (PCSK9is) who initiated treatment within the first 6 months of market availability.

Patients and methods: This retrospective cohort study used IQVIA's longitudinal open-source point-of-sale pharmacy claims database (LRx) and PharMetrics Plus (P+) health plan claims database to identify patients initiating a PCSK9i between January 1, 2016 and June 30, 2016. The index date was defined as the date of the first PCSK9i prescription (index claim) during the enrollment window; patients were followed for $\geq 6$ months postindex. Patient characteristics including use of baseline lipid-lowering therapy (LLT) and measures such as persistence and adherence to PCSK9i therapy were evaluated with respect to health plan type (commercial vs Medicare). Results: Overall, patients initiating PCSK9i $(n=13,151)$ had a mean age of 66 years, and $51 \%$ were male. Approximately $67.4 \%$ of patients used some form of LLT (statin and/or ezetimibe) in the 24 months prior to initiating PCSK9i therapy. The proportion of patients covered by a commercial health plan (51.2\%) was similar to that covered by Medicare (48.8\%). Persistence on PCSK9i was marginally longer for patients with commercial insurance than Medicare (mean days on therapy 202.2 vs 198.5 ). Overall, $42.6 \%$ of patients discontinued their PCSK9i during the 180 days of follow-up.

Conclusion: This study demonstrates that a large proportion of patients discontinue PCSK9i therapy at 30 and 90 days, which are the time frames for which many health plans require recertification to continue access to PCSK9i. Future studies looking at treatment patterns among patients who initiate PCSK9i therapy after the first 180 days once health plan formularies and utilization management criteria were finalized are needed to understand more comprehensively real-world PCSK9i usage patterns.

Keywords: LDL-C, statin, atherosclerotic cardiovascular disease, ASCVD, heterozygous familial hypercholesterolemia, $\mathrm{HeFH}$, lipid lowering

\section{Introduction}

Treatment for patients with heterozygous familial hypercholesterolemia $(\mathrm{HeFH})$ and atherosclerotic cardiovascular disease (ASCVD) who have elevated low-density lipoprotein cholesterol (LDL-C) typically includes HMG-CoA reductase inhibitors (statins), bile acid sequestrants, fibrates, and nicotinic acid (niacin) as first-line therapy. ${ }^{1-3}$ However, many patients cannot reach optimal LDL-C goal with statin therapy alone or are intolerant to statins due to adverse events ${ }^{4,5}$ and require additional treatment that often includes ezetimibe and proprotein convertase subtilisin/kexin type nine inhibitors (PCSK9is). ${ }^{6,7}$ 
PCSK9is have been found to play an important role in regulating cholesterol levels by binding to LDL/LDL-C receptor complex and promoting the elimination of LDL-C. ${ }^{8-11}$ The PCSK9is evolocumab and alirocumab were approved by the Food and Drug Administration in 2015 for use in patients with HeFH or ASCVD along with diet and maximally tolerated statin therapy, who are unable to achieve LDL-C goals despite maximal tolerated lipid-lowering therapy (LLT) and proper diet. ${ }^{12,13}$ In December 2017, the approved indications for evolocumab were expanded to include reductions in risk for myocardial infarction, stroke, and coronary revascularization in adults with established cardiovascular disease based on results from the Further Cardiovascular Outcomes Research with PCSK9 Inhibition in Subjects with Elevated Risk (FOURIER) outcomes study. ${ }^{12}$ The FOURIER trial of evolocumab demonstrated a $15 \%-20 \%$ relative reduction in major cardiovascular events vs placebo in patients with ASCVD and other cardiovascular risk factors, leading to updates in clinical and scientific guidelines. ${ }^{14}$ In a meta-analysis of clinical trial data, Li et al reported significant decreases in LDL-C of $\sim 65.3 \mathrm{mg} / \mathrm{dL}$ among patients treated with a PCSK9i. ${ }^{9}$ A separate meta-analysis conducted by Zhang et al among patients treated with statin therapy found that biweekly administration of $140 \mathrm{mg}$ of evolocumab and monthly treatment of $420 \mathrm{mg}$ both reduced LDL-C by $>50 \%$ vs placebo, with the biweekly administration having the greatest reduction $(-60.4 \%)$ after 12 weeks of treatment. ${ }^{15}$ Biweekly treatment with $50-150 \mathrm{mg}$ alirocumab resulted in LDL-C reductions slightly over $50 \%$ vs placebo. ${ }^{15}$ No significant difference in the incidence of treatment-emergent adverse events was observed in patients who received PCSK9i compared with controls who received placebo with or without other LLT. ${ }^{9,16}$

Although there are numerous studies providing efficacy and safety information on PCSK9is from clinical trials, ${ }^{16-18}$ real-world evidence on treatment patterns with PCSK9is within their first year of market availability in the United States is lacking. Therefore, the goal of this retrospective analysis was to describe demographic and clinical characteristics of patients initiating PCSK9is within the first 180 days of market availability.

\section{Patients and methods}

This was a retrospective cohort study using data on commercially insured and Medicare patients initiating PCSK9i therapy with evolocumab or alirocumab between January 1, 2016 and June 30, 2016. PCSK9i-dispensing patterns including dose titration and utilization of other LLTs including statin and/or ezetimibe prior to initiating PCSK9is were evaluated in these patients. The current study also evaluated treatment patterns (including persistence, adherence, discontinuation, and reinitiation) of evolocumab, alirocumab, and statin and/or ezetimibe therapy.

\section{Data source}

This study involved the use of two databases: IQVIA's open-source point-of-service longitudinal pharmacy claims database (LRx) and PharMetrics Plus ( $\mathrm{P}+$ ) health plan claims database. LRx captures information on adjudicated dispensed prescriptions sourced from retail, mail, long-term care, and specialty pharmacies. This database provides around $88 \%$ coverage of the retail channel, 55\% coverage of standard mail service, and $40 \%-70 \%$ coverage of specialty pharmacy volume and was used to evaluate treatment patterns, PCSK9i patient characteristics and dispensing patterns, and utilization of other LLTs.

As a pharmacy claims database, clinical and diagnostic information is not available in LRx. Thus, to better understand the clinical characteristics and prevalence of ASCVD in the study population, the LRx database was linked to $\mathrm{P}+$ and the subset of linked patients was analyzed. The aggregated $\mathrm{P}+$ database comprises adjudicated claims for more than 150 million unique enrollees across the United States with diverse representation of geography, employers, payers, providers and therapy areas. $\mathrm{P}+$ database has detailed information on inpatient/outpatient diagnoses and procedures and is representative of the national, commercially insured population for individuals aged 65 years and below.

All data were compliant with the Health Insurance Portability and Accountability Act to protect patients' privacy.

\section{Patient selection}

This study included patients with evidence of at least one newly dispensed prescription for a PCSK9i (evolocumab or alirocumab) between January 1, 2016 and June 30, 2016. The index date was defined as the date of the first PCSK9i prescription fill observed within this time window (Figure 1). The pharmacy dispensing the index prescription was required to have consistently submitted claims to the LRx database throughout the study period to maximize the likelihood that subsequent fills would be captured. In addition, patients were required to be 18 years of age or older on the index date and have no prescription claim for evolocumab or alirocumab prior to the index date. Patients with missing information on age, gender, or prescription were not included. Patients were followed for a minimum of 6 months postindex (Figure 1). 


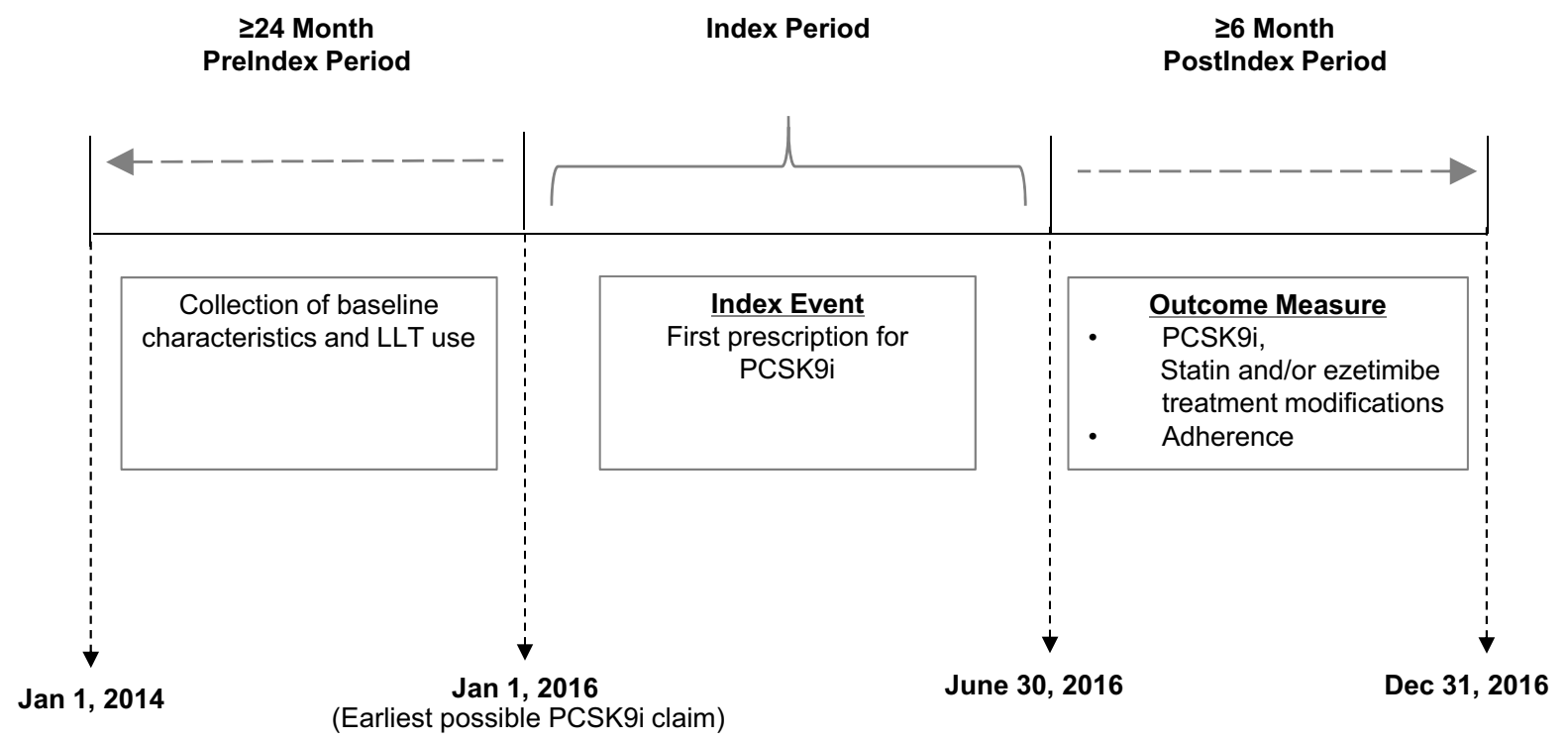

Figure I Study design.

Abbreviations: LLT, lipid-lowering therapy; PCSK9i, proprotein convertase subtilisin/kexin type nine inhibitor.

A subgroup of patients that linked to the $\mathrm{P}+$ database was evaluated for an ASCVD diagnosis prior to the PCSK9i index date. The study period included a 24-month preindex period to evaluate patient's baseline characteristics and a minimum of 6-month postindex follow-up period to evaluate the other outcome measures.

This study was based on deidentified claims data, which do not meet the definition of human subject research and thus are not subject to human subject regulation.

\section{Measures of outcomes}

\section{Demographic and clinical characteristics of early} initiators of PCSK9i therapy

Patient demographics (including age, gender, and geographic region) were assessed on the index date overall and by payer types (commercial vs Medicare). Prescribing physician specialty was recorded based on the index PCSK9i claim. Preselected concomitant medications (antidiabetic agents, antihypertensive agents, anticoagulants, antiarrhythmic agents, LLT) were collected during the 12-month period prior to the index date.

Baseline LLT was recorded during 6-month time intervals over a 24-month period prior to the index date and categorized as no statin and/or ezetimibe, statin only (subdivided into low-, moderate-, and high-intensity statin use based on the statin claim closest to but before the index date), ezetimibe monotherapy, and ezetimibe + statin therapy. Patients recorded as receiving ezetimibe + statin therapy included those receiving ezetimibe-statin dual therapy (a single NDC for the combination) and ezetimibe + statin combination therapy (separate NDCs for ezetimibe and a statin each), with at least 1 day overlap in days' supply. LLT use postindex was also described.

History of ASCVD-related diagnosis was captured in the 12 months prior to the index date using the presence of diagnosis codes for peripheral artery disease, coronary artery disease, angina, acute myocardial infarction, or stroke/ transient ischemic attack or CPT procedure codes for percutaneous coronary intervention, coronary artery bypass graft, or angioplasty on any medical claim, or by a pharmacy claim for antiplatelet therapy for those without an ASCVD medical claim. ASCVD history was assessed as the proportion of patients with indication of an ASCVD event.

\section{PCSK9i treatment patterns}

PCSK9i treatment patterns were measured as persistence, adherence, and treatment switch. Persistence with PCSK9i was assessed as the number of days on therapy from the index date until the end of follow-up or the end of days' supply of the last prescription fill. A gap of 60 days or more between the last day of days' supply of one prescription and the start of the next prescription was considered as discontinuation of therapy and thus the end of the persistent period. Adherence to PCSK9i was assessed as proportion of days covered (PDC; measured as the number of distinct days with treatment in the follow-up period divided by the number of days in the 
follow-up period) and medication possession ratio (MPR; measured as the sum of days of treatment supply divided by time on therapy [ie, the persistence period]).

\section{Statin and/or ezetimibe treatment patterns}

Adherence to statin and/or ezetimibe during the preindex period was assessed, as was the proportion of patients with statin and/or ezetimibe during the preindex period who had a postindex treatment modification (discontinuation or augmentation) to their statin and/or ezetimibe regimen. Among all patients with statin and/or ezetimibe observed during the preindex period and overlapping with the PCSK9i index date (thus, were receiving PCSK9i along with other LLT), statin and/or ezetimibe treatment patterns were assessed as the proportion of patients who discontinue, reinitiate, augment, or have intensity change of preindex statin and/or ezetimibe therapy any time during the postindex period. Discontinuation of statin and/or ezetimibe was defined in the same way as that of PCSK9i. Reinitiation was defined as the observation of a statin and/or ezetimibe prescription after a gap of more than 60 days without therapy. Augmentation was defined as the addition of a statin to ezetimibe or the addition of ezetimibe to a statin. Statin intensity changes were assessed as switching from one intensity level to a different intensity level (as defined in Table S1) among patients with a prescription for statin in the preindex period.

\section{Statistical analyses}

No statistical comparisons were performed; all data are presented descriptively. Kaplan-Meier curves were generated to assess time to discontinuation of PCSK9i therapy.

\section{Results}

\section{Patient and clinical characteristics}

After applying inclusion and exclusion criteria, a total of 13,151 PCSK9i initiators were identified (6,771 evolocumab and 6,380 alirocumab). Patient baseline characteristics are shown in Table 1 . The mean (SD) age of the study population was 66 (10.6) years. There were slightly more males $(51.2 \%)$ than females, and slightly more patients were insured by a commercial payer (51.2\%) than Medicare (48.8\%).

The majority of the initial PCSK9i prescriptions were written by cardiologists $(57.4 \%$ vs $32.0 \%$ from primary care providers). This was expected as many of the health plans during the early period of market availability of PCSK9i's require a cardiologist to consult or prescribe the agents for payment approval. In the 12 months prior to initiating a PCSK9i, antihypertensive therapy was used by most $(72.4 \%)$ of the patients, anticoagulants were used by $36.7 \%$, and antidiabetic drugs were used by $25.7 \%$ (Table 1 ).

Use of any baseline LLT in the form of statin and/or ezetimibe therapy any time during the 6 months prior to initiating PCSK9i was observed in only $45.2 \%$ of patients. However, when the preindex period was increased to 24 months, baseline LLT utilization increased to $67.4 \%$. A history of statin and/or ezetimibe monotherapy increased from $26.1 \%$ to $42.3 \%$ and from $10.1 \%$ to $14.4 \%$, respectively, when the preindex period was increased from 6 to 24 months. Use of statin and ezetimibe combination therapy increased from $9.0 \%$ to $10.7 \%$ over the 24 -month period (Figure 2 ). There was a general trend for a higher proportion of patients enrolled in a commercial health care plan to use statin and ezetimibe combination therapy or ezetimibe monotherapy, whereas a greater portion of patients with Medicare used statins only (Table 2).

\section{PCSK9i treatment patterns}

The initial dose for all patients initiating evolocumab was $140 \mathrm{mg}$ every 2 weeks. For patients initiating therapy with alirocumab, $88.3 \%$ started on the $75 \mathrm{mg}$ every 2 weeks dose and $11.7 \%$ received $150 \mathrm{mg}$ every 2 weeks. Postindex dose changes were observed in only $3.5 \%$ of all patients. Dose changes were observed more frequently among patients with Medicare (59.3\%) than a commercial payer (40.7\%).

Patients were persistent with PCSK9is for a mean (SD) of 201.6 (1.0) days. Mean (SD) days on PCSK9i therapy was longer for patients with a commercial payer (202.2 [1.4]) than Medicare (198.5 [1.5]). A large proportion of patients were found to discontinue PCSK9i therapy at 30 and 90 days (Figure 3). Discontinuation of PCSK9i was observed in $42.6 \%$ of patients and was similar between patients with commercial vs Medicare payers (42.5\% vs 42.6\%, respectively). Reasons for discontinuation are unknown but could have been due to poor tolerability, noncompliance, not willing to perform injections, and insurance or cost issues. Of the patients who discontinued, $9.1 \%$ reinitiated therapy $(8.4 \%$ with a commercial payer and $9.8 \%$ with Medicare).

Mean (SD) PDC was 0.64 (0.33), with $48.9 \%$ of patients having PDC $\geq 0.80$. Mean (SD) PDC was similar between patients with a commercial payer $(0.65$ [0.33]) and Medicare $(0.63[0.34])$.

\section{Statin and/or ezetimibe treatment patterns} There were a total of 4,038 patients $(30.7 \%)$ using statin and/ or ezetimibe at the time they were initiated on a PCSK9i. Among the statin-only users, a majority (60.6\%) had no 
Table I Characteristics of early initiators of PCSK9i therapy by payer type

\begin{tabular}{|c|c|c|c|}
\hline Baseline characteristics & $\begin{array}{l}\text { Total } \\
(\mathrm{N}=|3,15|)\end{array}$ & $\begin{array}{l}\text { Commercial insurance } \\
(\mathrm{N}=6,730)\end{array}$ & $\begin{array}{l}\text { Medicare insurance } \\
(\mathrm{N}=6,42 \mathrm{I})\end{array}$ \\
\hline \multicolumn{4}{|l|}{ Age group (years), \% } \\
\hline $18-34$ & 0.9 & 1.3 & 0.5 \\
\hline $35-44$ & 2.6 & 3.9 & 1.2 \\
\hline $45-54$ & 10.5 & 16.3 & 4.3 \\
\hline $55-64$ & 25.4 & 38.6 & 11.5 \\
\hline$\geq 65$ & 60.7 & 39.9 & 82.5 \\
\hline \multicolumn{4}{|l|}{ Age } \\
\hline Mean (SD) & $66(10.6)$ & $62.1(10.6)$ & $70(8.9)$ \\
\hline Median & 67 & 62 & 71 \\
\hline \multicolumn{4}{|l|}{ Gender, \% } \\
\hline Male & 51.2 & 56.9 & 45.4 \\
\hline Female & 48.8 & 43.2 & 54.7 \\
\hline \multicolumn{4}{|l|}{ Geographic region, \% } \\
\hline Northeast & 18.3 & 16.3 & 20.3 \\
\hline Midwest & 26.0 & 18.8 & 33.6 \\
\hline South & 45.5 & 55.2 & 35.2 \\
\hline West & 10.1 & 9.6 & 10.5 \\
\hline Unknown & $0.2 \%$ & $0.1 \%$ & $0.3 \%$ \\
\hline \multicolumn{4}{|l|}{ Preindex medication use, $\%$} \\
\hline Antidiabetic drugs & 25.7 & 22.9 & 28.5 \\
\hline Antihypertensive drugs & 72.4 & 69.1 & 75.9 \\
\hline Anticoagulants & 36.7 & 33.7 & 39.8 \\
\hline Antiarrhythmic drugs & $\mathrm{I} . \mathrm{I}$ & 0.9 & 1.3 \\
\hline \multicolumn{4}{|c|}{ Index therapy prescriber specialty, \% } \\
\hline Cardiology & 57.4 & 57.1 & 57.8 \\
\hline Primary care $^{\mathrm{a}}$ & 32.0 & 33.6 & 30.4 \\
\hline Other/unknown & 10.6 & 9.4 & 11.9 \\
\hline
\end{tabular}

Note: ${ }^{a}$ Includes internal medicine and family practice physicians.

Abbreviation: PCSK9i, proprotein convertase subtilisin/kexin type nine inhibitor.

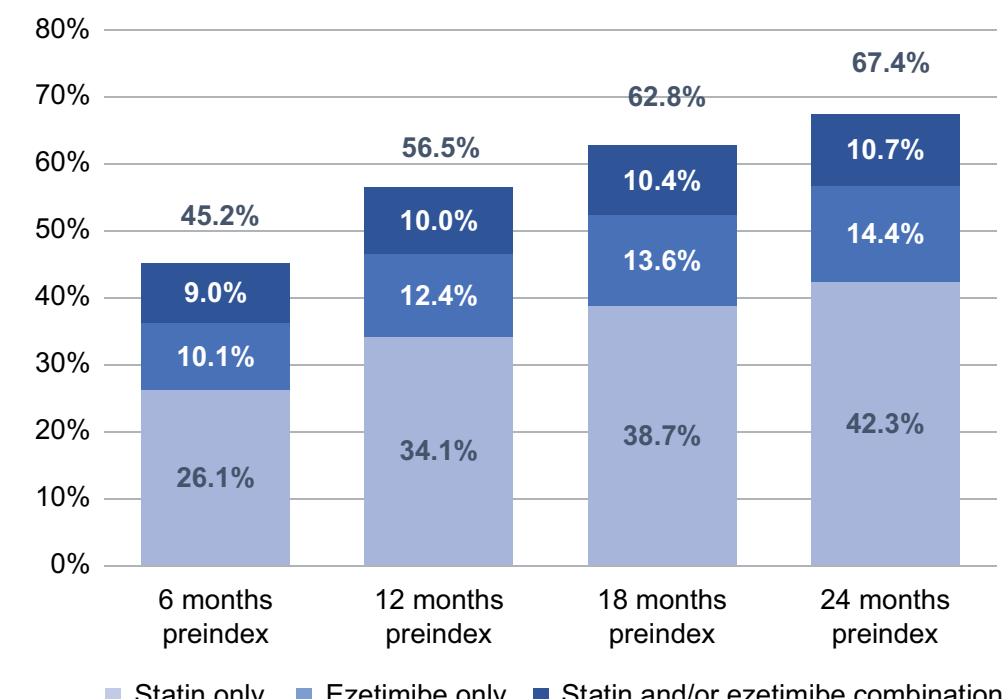

Figure 2 Baseline lipid-lowering therapy utilization among early initiators of PCSK9i therapy.

Abbreviation: PCSK9i, proprotein convertase subtilisin/kexin type nine inhibitor.

modification to their statin regimen, $35 \%$ discontinued their statin (with $13.1 \%$ reinitiating the statin), $2.5 \%$ had a change in statin intensity, and $2.0 \%$ augmented their statin with ezetimibe during the 6-month postindex period. Among those who used ezetimibe only, a majority $(65.8 \%)$ had no modification to their ezetimibe during postindex, $30.2 \%$ discontinued 
Table 2 Baseline lipid-lowering therapy use among early initiators of PCSK9i therapy by payer type

\begin{tabular}{|c|c|c|c|}
\hline Baseline lipid-lowering therapy & $\begin{array}{l}\text { Total } \\
(N=13,|5|)\end{array}$ & $\begin{array}{l}\text { Commercial insurance } \\
(\mathrm{N}=6,730)\end{array}$ & $\begin{array}{l}\text { Medicare insurance } \\
(\mathrm{N}=6,421)\end{array}$ \\
\hline \multicolumn{4}{|c|}{ Preindex statin and/or ezetimibe use 6 months preindex, $\%$} \\
\hline Any lipid-lowering therapy & 45.2 & 47.7 & 42.6 \\
\hline Statin therapy only & 26.1 & 25.9 & 26.2 \\
\hline Low-intensity statin & 3.3 & 3.2 & 3.4 \\
\hline Medium-intensity statin & 11.8 & 11.3 & 12.4 \\
\hline High-intensity statin & 10.9 & 11.4 & 10.5 \\
\hline Ezetimibe only & 10.1 & 10.8 & 9.5 \\
\hline Statin and/or ezetimibe combination & 9.0 & 11.0 & 7.0 \\
\hline Low-intensity statin & 0.7 & 0.8 & 0.7 \\
\hline Medium-intensity statin & 2.8 & 3.2 & 2.4 \\
\hline High-intensity statin & 5.5 & 7.0 & 3.4 \\
\hline \multicolumn{4}{|c|}{ Preindex statin and/or ezetimibe use 12 months preindex, $\%$} \\
\hline Any lipid-lowering therapy & 56.5 & 58.6 & 54.3 \\
\hline Statin therapy only & 34.1 & 33.6 & 34.7 \\
\hline Low-intensity statin & 13.6 & 13.7 & 13.6 \\
\hline Medium-intensity statin & 47.7 & 45.9 & 49.5 \\
\hline High-intensity statin & 38.7 & 40.4 & 37.0 \\
\hline Ezetimibe only & 12.4 & 13.0 & 11.8 \\
\hline Statin and/or ezetimibe combination & 10.0 & 12.0 & 7.9 \\
\hline Low-intensity statin & 8.5 & 7.7 & 9.9 \\
\hline Medium-intensity statin & 32.5 & 30.4 & 35.8 \\
\hline High-intensity statin & 59.0 & 62.0 & 54.3 \\
\hline \multicolumn{4}{|c|}{ Preindex statin and/or ezetimibe use 18 months preindex, $\%$} \\
\hline Any lipid-lowering therapy & 62.8 & 64.7 & 60.8 \\
\hline Statin therapy only & 38.7 & 38.1 & 39.4 \\
\hline Low-intensity statin & 5.5 & 5.5 & 5.6 \\
\hline Medium-intensity statin & 18.9 & 17.9 & 19.9 \\
\hline High-intensity statin & 14.3 & 14.7 & 14.0 \\
\hline Ezetimibe only & 13.6 & 14.0 & 13.1 \\
\hline Statin and/or ezetimibe combination & 10.4 & 12.5 & 8.3 \\
\hline Low-intensity statin & 0.9 & 1.0 & 0.9 \\
\hline Medium-intensity statin & 3.5 & 3.9 & 3.0 \\
\hline High-intensity statin & 6.1 & 7.6 & 4.4 \\
\hline \multicolumn{4}{|c|}{ Preindex statin and/or ezetimibe use 24 months preindex, \% } \\
\hline Any lipid-lowering therapy & 67.4 & 69.20 & 65.5 \\
\hline Statin therapy only & 42.3 & 41.50 & 43.2 \\
\hline Low-intensity statin & 6.2 & 6.20 & 6.3 \\
\hline Medium-intensity statin & 21.0 & 19.90 & 22.1 \\
\hline High-intensity statin & 15.1 & 15.30 & 14.8 \\
\hline Ezetimibe only & 14.4 & 14.90 & 13.8 \\
\hline Statin and/or ezetimibe combination & 10.7 & 12.80 & 8.6 \\
\hline Low-intensity statin & 0.9 & 1.00 & 0.9 \\
\hline Medium-intensity statin & 3.6 & 4.10 & 3.2 \\
\hline High-intensity statin & 6.2 & 7.80 & 4.5 \\
\hline
\end{tabular}

Abbreviation: PCSK9i, proprotein convertase subtilisin/kexin type nine inhibitor.

ezetimibe (with $11.4 \%$ reinitiating therapy), and $4.0 \%$ augmented their ezetimibe with a statin. Among patients with statin and/or ezetimibe combination therapy, $44.9 \%$ had no modification postindex, $21.7 \%$ discontinued the statin, $20.6 \%$ discontinued the ezetimibe, $11.7 \%$ discontinued both therapies, and $1.1 \%$ had a change in statin intensity.

\section{History of ASCVD}

Overall, of the 514 patients evaluated in the linked LRx $+\mathrm{P}+$ dataset, nearly half (47.1\%) had an ASCVD-related diagnosis or procedure at some point during the preindex period. An additional $5.9 \%$ of patients were found to have used antiplatelet therapy, which is a measure suggestive of ASCVD history. 


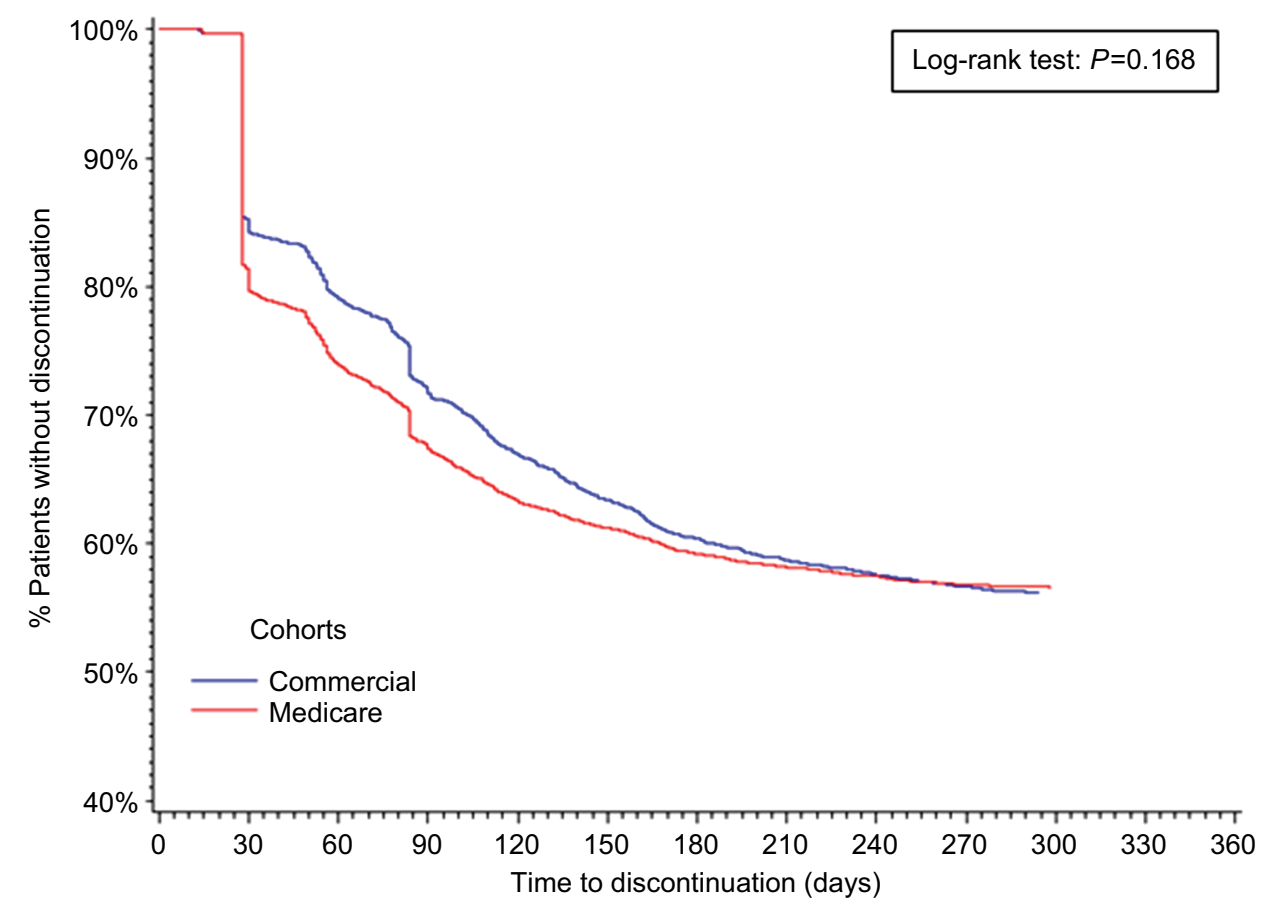

Figure 3 Time to discontinuation of index PCSK9i among early initiators of PCSK9i therapy, by payer type.

Abbreviation: PCSK9i, proprotein convertase subtilisin/kexin type nine inhibitor.

\section{Discussion}

To date, no other studies have assessed the characteristics and treatment patterns of patients initiating therapy on PCSK9is. This study showed that early initiators of PCSK9is are generally older ( $>65$ years of age), relatively balanced by gender, and that a slightly higher proportion are covered by a commercial health plan vs Medicare. Not surprisingly, given health plans' prior authorization requirements, cardiologists are more likely than any other health care provider to start a patient on a PCSK9i. In their study of PCSK9i approval and rejection rates, Hess et al reported that age $>65$ years and having a prescription from a cardiologist or nonprimary care provider were significantly associated with PCSK9i approval. ${ }^{19}$ Prescribers tend to start patients on a dosing regimen that is consistent with prescribing recommendations, as all of the evolocumab patients were initiated on the $140 \mathrm{mg}$ every 2 weeks dose, and the majority of alirocumab patients were initiated on the $75 \mathrm{mg}$ every 2 weeks dose. Patients who remain on their PCSK9i tend to maintain their initial dose, with $<5 \%$ of patients experiencing a dose change.

Increasing the preindex period from 6 to 24 months increased the percentage of patients with a trial of statin and/ or ezetimibe therapy from $45.2 \%$ to $67.4 \%$. It is not known if this would continue to increase if the preindex period were further increased. However, since the majority of Utilization
Management criteria at this time required patients to either be statin intolerant or currently taking a statin, it is likely that an even longer lookback period may be necessary to fully observe all prior statin use. The observation that at least $33 \%$ of patients were not using a statin nor ezetimibe at a given time could also suggest that, for these patients, statin and/or ezetimibe therapy may have been ineffective at controlling LDL-C; alternatively, the allure of the PSCK9is may have caused physicians to prescribe them for patients prior to assessing the response to the highest tolerable dose of a statin and/or ezetimibe. The majority of patients $(>65 \%)$ who were on a statin and/or ezetimibe at the time of starting the PCKS9i remained on their statin and/ or ezetimibe throughout our observation period.

Persistence with LLT is known to be suboptimal, ${ }^{20}$ and this was further observed in this study with PCSK9is. Overall, patients remained on therapy for about 6.5 months. This study demonstrates that a large proportion of patients discontinue PCSK9i therapy at 30 and 90 days, which are the time frames for which many health plans require recertification to continue access to PCSK9i. This study also showed that discontinuation rates were similar between patients with commercial and Medicare coverage, suggesting that patients' health plan have little influence on persistence with PCSK9i. It is, however, plausible that despite the payer, changes in health plans and reimbursement rates could lead a patient to 
no longer refill their therapy, though more research is needed to better understand why patients discontinue their PCSK9i.

The data presented here also suggest that improvements with adherence to PCSK9is are needed. Although Saborowski et al indicated that 27 out of the 31 patients they surveyed $(87 \%)$ were adherent with their PCSK9i, ${ }^{20}$ the patients observed in the current study had their PCSK9i available (on average) $64 \%$ of the time, with only $48.9 \%$ of them having therapy coverage at least $80 \%$ of the time. The adherence estimates reported by Saborowski et $\mathrm{al}^{21}$ were obtained by a survey on which patients were asked if they injected the medication as prescribed, which is subject to biased reporting. Our estimates were derived from actual prescription fill data, which is not influenced by recall and allows for better approximations of gaps in therapy.

Results of this study should be considered in the context of the following limitations. First, this analysis was retrospective in nature and relied upon coding used for billing to make inferences about the measures of interest. No causal relationships can be established from this observational study. Second, this research employed the use of an open-source pharmacy claims database. Only pharmacies who continuously submitted claims to the database were used for this study, creating the potential for information bias. Third, evolocumab and alirocumab are primarily distributed through specialty pharmacies, and there was limited capture of claims in LRx that came through this channel around the initial product availability period; therefore, this study does not report all PCSK9i utilization. Also, as LRx is a pharmacy claims database, no diagnostic information is available, making it unfeasible to confirm a diagnosis of $\mathrm{HeFH}$ or ASCVD in association with the PCSK9i prescription in LRx alone. By linking the LRx claims to a health care claims database $(\mathrm{P}+)$, we were able to confirm an ASCVD diagnosis in nearly half of the patients who remained in the linked cohort. Additionally, laboratory data such as lipid concentrations are not available in the pharmacy claims database.

\section{Conclusion}

This study provides insight into the characteristics and treatment patterns of patients initiating a new-to-market therapy (PCSK9i). This study elucidates some of the challenges with persistence and adherence with the novel PCSK9is, while highlighting useful information in terms of their dispensing and dosing patterns, as well as the use of other LLTs prior to the PCSK9i. The data used for this study were collected within the first year of market availability, during which time there were uncertainties around formulary decisions. Additionally, patients' and providers' perspectives on these therapies as well as exposure time were limited. Future studies looking at treatment patterns among patients who initiate PCSK9i therapy after the first 180 days once health plan formularies and utilization management criteria were finalized are needed to understand more comprehensively real-world PCSK9i usage patterns.

\section{Acknowledgment}

The authors would like to thank Yao Ding, PhD for her contributions throughout the execution of this study and Kainan Sun, $\mathrm{PhD}$ for analytic support.

\section{Disclosure}

This research was sponsored by Amgen.

Dionne M Hines and Rolin L Wade are employees of IQVIA; Amgen hired IQVIA to conduct this study. Pallavi Rane, Jeetvan Patel, and David J Harrison are employees and shareholders of Amgen. The authors report no other conflicts of interest in this work.

\section{References}

1. The Familial Hypercholestrolemia Foundation. Available from: https:// thefhfoundation.org/heterozygous-vs-homozygous-fh. Accessed May 12, 2017.

2. Stone NJ, Robinson JG, Lichtenstein AH. ACC/AHA guideline on the treatment of blood cholesterol to reduce atherosclerotic cardiovascular risk in adults: a report of the American College of Cardiology/American Heart Association Task Force on Practice Guidelines. J Am Coll Cardiol. 2013;2014(63):2889-2934.

3. Reiner $\breve{Z}$. Management of patients with familial hypercholesterolaemia. Nat Rev Cardiol. 2015;12(10):565.

4. Imić I, Reiner Ž. Adverse effects of statins - myths and reality. Curr Pharm Des. 2015;21(9):1220-1226.

5. Reiner Z. Resistance and intolerance to statins. Nutr Metab Cardiovasc Dis. 2014;24(10):1057-1066.

6. Dadu RT, Ballantyne CM. Lipid lowering with PCSK9 inhibitors. Nat Rev Cardiol. 2014;11(10):563-575.

7. Karalis DG, Victor B, Ahedor L, Liu L. Use of lipid-lowering medications and the likelihood of achieving optimal LDL-cholesterol goals in coronary artery disease patients. Cholesterol. 2012;2012:1-7.

8. Lepor NE, Kereiakes DJ. The PCSK9 inhibitors: a novel therapeutic target enters clinical practice. Am Health Drug Benefits. 2015;8(9):483-488.

9. Li C, Lin L, Zhang W, et al. Efficiency and safety of proprotein convertase subtilisin/kexin 9 monoclonal antibody on hypercholesterolemia: a meta-analysis of 20 randomized controlled trials. J Am Heart Assoc. 2015;4(6):e001937.

10. Pećin I, Hartgers ML, Hovingh GK, Dent R, Reiner Ž. Prevention of cardiovascular disease in patients with familial hypercholesterolaemia: the role of PCSK9 inhibitors. Eur J Prev Cardiol. 2017;24(13):1383-1401.

11. Reiner Z̆. PCSK9 inhibitors in clinical practice: expectations and reality. Atherosclerosis. 2018;270:187-188.

12. Repatha prescribing information. Available from: http://pi.amgen.com/ united_states/repatha/repatha_pi_hcp_english.pdf. Accessed August 23, 2017.

13. Praluent prescribing information. Available from: http://products.sanofi. us/praluent/praluent.pdf. Accessed August 23, 2017.

14. Sabatine MS, Giugliano RP, Keech AC, et al. Evolocumab and clinical outcomes in patients with cardiovascular disease. $N$ Engl J Med. 2017;376(18):1713-1722. 
15. Zhang XL, Zhu QQ, Zhu L, et al. Safety and efficacy of anti-PCSK9 antibodies: a meta-analysis of 25 randomized, controlled trials. BMC Med. 2015;13:123.

16. Robinson JG, Farnier M, Krempf M, et al. Efficacy and safety of alirocumab in reducing lipids and cardiovascular events. $N$ Engl J Med. 2015;372(16):1489-1499.

17. Blom, DJ, Hala T, Bolognese M, et al. A 52-week placebocontrolled trial of evolocumab in hyperlipidemia. $N$ Engl J Med. 2014;370(19):1809-1819.

18. Schwartz GG, Bessac L, Berdan LG, et al. Effect of alirocumab, a monoclonal antibody to PCSK9, on long-term cardiovascular outcomes following acute coronary syndromes: rationale and design of the ODYSSEY outcomes trial. Am Heart J. 2014;168(5):682-689.
19. Hess GP, Natarajan P, Faridi KF, Fievitz A, Valsdottir L, Yeh RW. Proprotein convertase subtilisin/kexin type 9 inhibitor therapy: payer approvals and rejections, and patient characteristics for successful prescribing. Circulation. 2017;136(23):2210-2219.

20. Foody JM, Joyce AT, Rudolph AE, Liu LZ, Benner JS. Persistence of atorvastatin and simvastatin among patients with and without prior cardiovascular diseases: a US managed care study. Curr Med Res Opin. 2008;24(7):1987-2000.

21. Saborowski M, Dölle M, Manns MP, Leitolf H, Zender S. Lipid-lowering therapy with PCSK9-inhibitors in the management of cardiovascular high-risk patients: effectiveness, therapy adherence and safety in a real world cohort. Cardiol J. 2018;25(1):32-41. 


\section{Supplementary material}

Table SI Statin intensity classification

\begin{tabular}{|c|c|c|c|c|}
\hline \multirow[t]{2}{*}{ Statin therapy } & \multicolumn{4}{|l|}{ Daily dose } \\
\hline & $\begin{array}{l}\text { Low intensity } \\
\text { (mg/day) }\end{array}$ & $\begin{array}{l}\text { Moderate intensity } \\
\text { (mg/day) }\end{array}$ & $\begin{array}{l}\text { High intensity } \\
\text { (mg/day) }\end{array}$ & $\begin{array}{l}\text { Notes (classification of } \\
\text { atypical doses) }\end{array}$ \\
\hline Atorvastatin & $<10$ & 10 to $<40$ & $\geq 40$ & $30 \mathrm{mg} /$ day = moderate intensity \\
\hline Fluvastatin & $<80$ & 80 & $\mathrm{n} / \mathrm{a}$ & $10 \mathrm{mg} /$ day = low intensity \\
\hline Lovastatin & $<40$ & $\geq 40$ & $\mathrm{n} / \mathrm{a}$ & $\begin{array}{l}10 \mathrm{mg} / \mathrm{day}=\text { low intensity } \\
80 \mathrm{mg} / \mathrm{day}=\text { moderate intensity }\end{array}$ \\
\hline Pitavastatin & $<2$ & $\geq 2$ & $\mathrm{n} / \mathrm{a}$ & \\
\hline Pravastatin & $<40$ & $\geq 40$ & $\mathrm{n} / \mathrm{a}$ & $<10 \mathrm{mg} /$ day $=$ low intensity \\
\hline Rosuvastatin & $<5$ & 5 to $<20$ & $\geq 20$ & $\begin{array}{l}<5 \mathrm{mg} / \text { day }=\text { low intensity } \\
15 \mathrm{mg} / \text { day }=\text { moderate intensity }\end{array}$ \\
\hline Simvastatin & $<20$ & 20 to $<80$ & $\geq 80$ & $\begin{array}{l}<20 \mathrm{mg} / \text { day }=\text { low intensity } \\
>40 \text { to }<80 \mathrm{mg} / \text { day = moderate } \\
\text { intensity } \\
\geq 80 \mathrm{mg} / \text { day = high intensity }\end{array}$ \\
\hline
\end{tabular}

\section{Publish your work in this journal}

Vascular Health and Risk Management is an international, peerreviewed journal of therapeutics and risk management, focusing on concise rapid reporting of clinical studies on the processes involved in the maintenance of vascular health; the monitoring, prevention and treatment of vascular disease and its sequelae; and the involvement of metabolic disorders, particularly diabetes. This journal is indexed on PubMed Central and MedLine. The manuscript management system is completely online and includes a very quick and fair peer-review system, which is all easy to use. Visit http://www.dovepress.com/ testimonials.php to read real quotes from published authors. 